\title{
Contribution of diuretic therapy with human serum albumin to the management of ascites in patients with advanced liver cirrhosis: A prospective cohort study
}

\author{
TORU NAKAMURA ${ }^{1,2}$, MICHIO SATA $^{1}$, KAZUMASA HIROISHI $^{3}$, NAOHIKO MASAKI $^{4}$, HISATAKA MORIWAKI $^{5}$, \\ YOSHIKAZU MURAWAKI $^{6}$, HIROSHI YATSUHASHI ${ }^{7}$, SHIGETOSHI FUJIYAMA $^{8}$ and MICHIO IMAWARI ${ }^{3}$ \\ ${ }^{1}$ Division of Gastroenterology, Department of Medicine, Kurume University School of Medicine; \\ ${ }^{2}$ Liver Cancer Division, Research Center for Innovative Cancer Therapy, Kurume University, \\ Kurume, Fukuoka 830-0011; ${ }^{3}$ Division of Gastroenterology and Hepatology, Department of Medicine, \\ Showa University School of Medicine, Shinagawa-ku, Tokyo 142-8666; ${ }^{4}$ Department of Gastroenterology, \\ National Center for Global Health and Medicine, Shinjuku-ku, Tokyo $162-8655 ;{ }^{5}$ Department of Gastroenterology, \\ Gifu University School of Medicine, Gifu 501-1194; ${ }^{6}$ Second Department of Internal Medicine, Faculty of Medicine, \\ Tottori University, Yonago, Tottori $683-8504 ;{ }^{7}$ Clinical Research Center, National Hospital Organization, \\ Nagasaki Medical Center, Omura, Nagasaki $856-8562 ;{ }^{8}$ Department of Gastroenterology and Hepatology, \\ NTT West Kyushu Hospital, Chuo-ku, Kumamoto 862-8655, Japan
}

Received August 22, 2013; Accepted December 19, 2013

DOI: $10.3892 / \mathrm{mco} .2014 .245$

\begin{abstract}
The number of available studies on the role of human serum albumin (HSA) in the treatment of cirrhotic ascites is currently limited. In this study, we aimed to investigated the parameters associated with diuretic therapy withHSA in patients with advanced cirrhotic ascites. The patient inclusion criteria were cirrhotic ascites and a serum albumin (Alb) concentration of $<3.5 \mathrm{~g} / \mathrm{dl}$. A total of 49 patients registered and 38 patients were ultimately included in this study. The enrolled patients were mainly treated with oral spironolactone and furosemide, which were not specified; the HSA amount was also not specified, although the administration period was set to a maximum of 7 days. Our results demonstrated that the administration of HSA significantly increased the serum levels of Alb [0.97 g/dl; two-sided $95 \%$ confidence interval (CI): 0.83-1.11 g/dl] and decreased body weight $(-2.24 \mathrm{~kg} ; 95 \% \mathrm{CI}:-3.06$ to $-1.43 \mathrm{~kg})$, hematocrit ratio $(0.96$; $95 \% \mathrm{CI}$ : $0.94-0.98)$ and plasma renin concentration (day 4; geometric mean fold change, -0.1528 ; 95\% CI: -0.2510 to -0.0545 ; log-transformed data) in patients with advanced cirrhotic ascites. The observed weight loss was found to be correlated with the total amount of HSA administered $(\mathrm{P}=0.0012)$, as indicated by the results of the multiple
\end{abstract}

Correspondence to: Dr Toru Nakamura, Division of Gastroenterology, Department of Medicine, Kurume University School of Medicine, 67 Asahi-machi, Kurume, Fukuoka 830-0011, Japan

E-mail: ntoru@med.kurume-u.ac.jp

Key words: human serum albumin, liver cirrhosis, ascites, diuretics linear regression analysis. In conclusion, this study confirmed the efficacy of HSA in patients with advanced cirrhotic ascites.

\section{Introduction}

The mechanisms underlying the development of ascites in patients with liver cirrhosis are associated with several factors, without reaching any definitive conclusions. The underfilling theory (1) suggests that water and sodium retention may be a secondary phenomenon that results from the formation of ascites and the decreased effective circulating blood volume. With the progression of liver cirrhosis, the sinusoidal hydrostatic pressure and lymph formation in the liver are increased; when lymph formation exceeds the amount of flow to lymphatic vessels, it is retained as ascites. This subsequently decreases the effective circulating blood volume and causes retention of water and sodium in the renal tubules through neurohumoral factors. According to the underfilling theory, patients with cirrhotic ascites are treated with a low-salt diet and rest, with the addition of diuretic treatment for patients with cirrhotic ascites (2). Diuretic therapy improves ascites and edema by actively excreting retained water and sodium. Recent guidelines published in Japan reported the administration of human serum albumin (HSA) to patients with diuretic-resistant cirrhotic ascites who did not show improvement following administration of a diuretic alone $(2,3)$. The main purpose of HSA administration in the treatment of cirrhotic ascites is to increase the effective circulating blood volume. A previous article on the 'proper use of albumin' in the Japanese Guidelines for Blood Product Use and Blood Transfusion Therapy Practice states that hypertonic albumin (Alb) may be used as a concomitant drug in the short-term (maximum of 1 week) treatment of diuretic-resistant ascites (3). 
The following actions of HSA were previously established: First, HSA was shown to prevent circulatory failure following abdominal paracentesis of a large amount of ascitic fluid. It was reported that 8-10 $\mathrm{g}$ of Alb administered per 1 liter of drainage can prevent circulatory failure (4). Second, HSA was shown to prevent renal failure due to spontaneous bacterial peritonitis. Sort et al (5) reported that patients administered antibiotics plus HSA exhibited a more significant improvement in renal function and survival compared to those who received antibiotics alone. Finally, HSA was shown to improve renal function when used with terlipressin for the treatment of hepatorenal syndrome. A recovery of renal function was reported following combination therapy with terlipressin and intravenous HSA (6). In light of this evidence, Gentilini et al (7) conducted a randomized, controlled trial to investigate the efficacy of HSA in patients with cirrhotic ascites. Their results demonstrated that the co-administration of diuretic and Alb resulted in a better control of all parameters, including disappearance of ascites, duration of hospital stay, recurrence of ascites and hospital readmission due to ascites exacerbation, compared to the use of diuretics alone. The Clinical Practice Guidelines for the Management of Liver Cirrhosis by the Japanese Society of Gastroenterology state that 'HSA is useful in treating ascites associated with liver cirrhosis' (2). However, there are certain limitations to using HSA in patients with cirrhotic ascites, including the high treatment cost and the risk of infectious disease transmission with HSA, as it is a derivative of human blood. Therefore, HSA therapy is not currently widely used in an international setting.

We conducted a phase II (multicenter, randomized, open-label, parallel-controlled) clinical trial of KD-294, a recombinant HSA, in patients with cirrhotic ascites, initiated in 2005. Our results suggested that there may be a specific factor that is correlated with the improving effect of HSA on cirrhotic ascites. In particular, it was demonstrated that HSA may exert additional effects on diuretic therapy in patients with high plasma renin concentration (PRC) (8). In the present observational cohort study, which was conducted following the completion of the phase II study mentioned above, we aimed to investigate each parameter considered to be associated with the treatment of cirrhotic ascites with diuretics and HSA.

\section{Materials and methods}

Study drug. HSA, the study drug, was derived from human plasma and is currently marketed in Japan. The use of diuretics and concomitant drugs was not specified, nor was the use of a low-salt diet.

Patients. The study population comprised hospitalized patients diagnosed with liver cirrhosis and ascites, with a serum Alb concentration of $<3.5 \mathrm{~g} / \mathrm{dl}$. Patients with severe renal disease (serum creatinine levels $\geq 2.0 \mathrm{mg} / \mathrm{dl}$ or proteinuria $\geq 3.5 \mathrm{~g} / \mathrm{day}$ ), patients scheduled to undergo drainage of ascites and patients considered unsuitable for HSA administration, were excluded from this study. The patients were recruited from the following: Kurume University Hospital, Showa University Hospital, Gifu University Hospital, Tottori University Hospital, Nagasaki Medical Center and NTT West Kyushu Hospital.
Informed consent and patient registration. Prior to enrollment, each patient provided written informed consent to the investigator or sub-investigators after receiving a sufficient explanation of the study protocol and objective. This study was reviewed and approved by the local Ethics Committee of each study site and conducted in accordance with the principles of the Declaration of Helsinki.

Study design. This study was conducted as a prospective cohort study between November, 2007 and January, 2009. The HSA dose was tailored to each patient's requirements for the treatment of ascites and the treatment duration was set to a maximum of 7 days, according to the Japanese Guidelines for Blood Product Use and Blood Transfusion Therapy Practice (3). The type of the diuretic administered was not specified, whereas oral administration and injection were both permitted. The recorded parameters included body weight, ascites severity, hematological tests [hematocrit (Ht)], laboratory serum test (serum Alb concentration) and endocrine tests [PRC and aldosterone (ALD)]. It was mandatory that these parameters were measured on days 1, 4 and 6. If HSA was used for $>5$ days, a measurement was also performed on the day after the last day of administration. PRC and ALD were measured by the Mitsubishi Chemical Medience Corporation (Tokyo, Japan). HSA safety was not investigated, since it was previously established and was not considered necessary as this study was conducted while HSA was in use.

Methods of analysis. The mean value and standard deviation (SD) at each observation time point for Alb concentration, body weight and other laboratory parameters, as well as the mean value and $95 \%$ confidence interval (CI) (two-sided) for the changes from baseline to each observation time point, were calculated. The Ht ratio was calculated as the change from baseline to the observation time point by using the value at day 1 as the denominator and the values at each observation time point as the numerators. Logarithmic transformation was applied for PRC and ALD and the values were analyzed with normal distribution. The frequencies for the category of ascites status and the proportion of change in that status were calculated at each observation time point. The descriptive statistics were calculated for the weight change from baseline to the observation time point, in order to investigate the effect of the HSA dose and diuretic use on weight change by stratifying the total amount of HSA and that of the diuretic. In addition, a multiple linear regression analysis was performed, using an objective variable, such as weight change from baseline, as well as explanatory variables, such as the total amount of HSA and diuretics administered and the values of PRC and ALD. All the statistical analyses were performed using SAS software, version 9.2, or JMP software, version 8 (SAS Institute Japan Ltd., Tokyo, Japan). Two-sided tests at the 0.05 level were considered to be statistically significant.

\section{Results}

Study population. Of the 49 registered patients, 11 were considered unsuitable for the analysis and were excluded from this study. The exclusion criteria were as follows: patients 


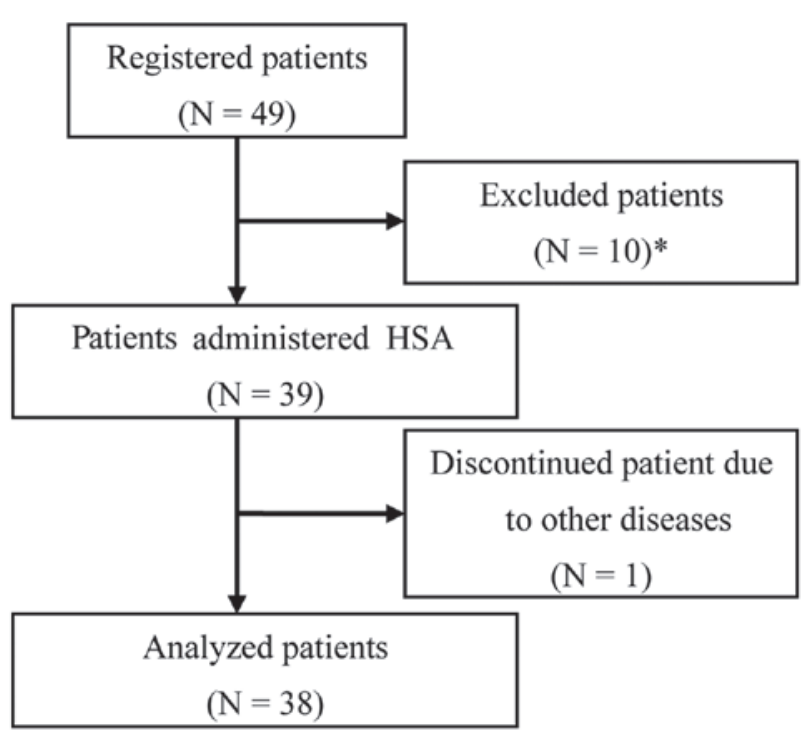

Figure 1. Flow diagram of the patient exclusion process. *The following patients were excluded: three patients with ascites due to suspected portal vein embolism, one patient with ascites due to diagnosed portal vein embolism who was administered human serum albumin (HSA) 1 week prior to the study initiation, two patients who were administered HSA 1 week prior to the study initiation and underwent abdominal paracentesis, three patients who received HSA 1 week prior to the study initiation and one patient who did not have ascites.

considered to have ascites due to portal vein embolism; patients with a history of abdominal paracentesis; patients who were administered HSA within 1 week prior to the study initiation; patients without ascites; and patients who discontinued treatment due to another disease (dementia) from day 2. The remaining 38 patients constituted the study cohort (Fig. 1) and their clinical characteristics are presented in Table I.

Use of HSA and diuretics. An HSA dose was administered in one or two vials per day (containing 12.5-25.0 g of HSA) for a maximum of 7 days. Four patients received a total dose of one vial of HSA for $\leq 3$ days $(37.5 \mathrm{~g}) ; 9$ patients received $>3$ but $<10$ vials $(>37.5$ but $<125.0 \mathrm{~g}$ ) and 25 patients received $\geq 10$ vials ( $\geq 125.0 \mathrm{~g})$.

As regards the use of diuretics, 28 patients received a diuretic for $\geq 7$ days prior to HSA administration and 10 patients received diuretics for $<7$ days or did not receive any diuretics prior to the initiation of HSA administration. As regards the use of anti-ALD diuretics, 23 patients received anti-ALD for $\geq 7$ days prior to the administration of HSA and 15 patients received anti-ALD for $<7$ days or not at all prior to the administration of HSA. The actual dose of diuretics was within the range recommended on the package insert.

HSA treatment decreases body weight, Ht ratio and PRC and increases serum Alb levels. The weight changes from baseline (day 1) were $-1.49 \mathrm{~kg}(95 \% \mathrm{CI}$ : -2.05 to -0.94$)$ on day 4 and $-2.24 \mathrm{~kg}$ (95\% CI: -3.06 to -1.43$)$ on day 6 (Table II). As regards the change in the ascites status, an improvement was observed in 14 of the 38 patients on day 4 and in 22 patients on day 6 (Table III).

As regards the change in laboratory values from baseline (day 1), the Alb concentration was significantly increased on
Table I. Clinical characteristics.

\begin{tabular}{|c|c|c|}
\hline Variables & Category & Patient no. $(\%)$ \\
\hline Gender & $\begin{array}{c}\text { Male } \\
\text { Female }\end{array}$ & $\begin{array}{l}24(63.2) \\
14(36.8)\end{array}$ \\
\hline $\begin{array}{l}\text { Age, years }(n=38) \\
\text { Mean } \pm \text { SD }\end{array}$ & $\begin{array}{c}<65 \\
66-74 \\
\geq 75 \\
66.1 \pm 11.0\end{array}$ & $\begin{array}{l}15(39.5) \\
13(34.2) \\
10(26.3)\end{array}$ \\
\hline $\begin{array}{l}\mathrm{BW}, \mathrm{kg}(\mathrm{n}=36) \\
\text { Mean } \pm \mathrm{SD}\end{array}$ & $\begin{array}{c}<50 \\
50-59 \\
60-69 \\
\geq 70 \\
59.37 \pm 15.36\end{array}$ & $\begin{array}{r}11(30.6) \\
9(25.0) \\
9(25.0) \\
7(19.4)\end{array}$ \\
\hline $\begin{array}{l}\text { Etiology of } \\
\text { liver cirrhosis }\end{array}$ & $\begin{array}{c}\text { HBV infection } \\
\text { HCV infection } \\
\text { ALC } \\
\text { Mixed }^{\mathrm{a}} \\
\text { Others }^{\mathrm{b}}\end{array}$ & $\begin{aligned} 5 & (13.2) \\
20 & (52.6) \\
5 & (13.2) \\
5 & (13.2) \\
3 & (7.9)\end{aligned}$ \\
\hline Alb, g/dl $(n=38)$ & $\begin{array}{c}<1.5 \\
1.5-1.9 \\
2.0-2.4 \\
2.5-2.9 \\
\geq 3.0 \\
2.29 \pm 0.34\end{array}$ & $\begin{aligned} & (0.0) \\
7 & (18.4) \\
20 & (52.6) \\
10 & (26.3) \\
1 & (2.6)\end{aligned}$ \\
\hline Ascites $^{c}$ & $\begin{array}{c}+ \\
2+ \\
3+\end{array}$ & $\begin{array}{r}8(21.1) \\
18(47.4) \\
12(31.6)\end{array}$ \\
\hline Edema & $\begin{array}{l}- \\
+\end{array}$ & $\begin{array}{l}13(34.2) \\
25(65.8)\end{array}$ \\
\hline Mean \pm SD & $\begin{array}{c}<25 \\
25-29 \\
30-34 \\
\geq 35 \\
30.23 \pm 4.55\end{array}$ & $\begin{array}{r}4(10.5) \\
14(36.8) \\
14(36.8) \\
6(15.8)\end{array}$ \\
\hline T-BIL, mg/dl (n=33) & $\begin{array}{c}\leq 1.2 \\
1.2-1.9 \\
2.0-2.9 \\
3.0-3.9 \\
4.0-4.9 \\
\geq 5.0 \\
2.18 \pm 1.38\end{array}$ & $\begin{array}{r}7(21.2) \\
11(33.3) \\
8(24.2) \\
3(9.1) \\
2(6.1) \\
2(6.1)\end{array}$ \\
\hline $\mathrm{Cr}, \mathrm{mg} / \mathrm{dl}(\mathrm{n}=36)$ & $\begin{array}{c}\leq 1.1 \\
1.1-1.9 \\
\geq 2.0 \\
0.899 \pm 0.354\end{array}$ & $\begin{array}{c}31(86.1) \\
5(13.9) \\
0(0.0)\end{array}$ \\
\hline BUN, mg/dl $(n=31)$ & $\begin{array}{c}\leq 23 \\
23-50 \\
>50 \\
19.95 \pm 9.82\end{array}$ & $\begin{aligned} 22 & (71.0) \\
9 & (29.0) \\
0 & (0.0)\end{aligned}$ \\
\hline
\end{tabular}

${ }^{a} \mathrm{HCV}$ LC and ALC: 1 patient; HBV LC and HCV LC, 2 patients; ALC and AIH, 1 patient; PBC, HBV LC and HCV LC, 1 patient. 'PBC, 1 patient; other liver cirrhosis, 2 patients. ${ }^{\mathrm{c}}$ Ascites status: + , mild; $2+$, moderate; $3+$, severe. BW, body weight; HBV, hepatitis B virus; $\mathrm{HCV}$, hepatitis C virus; ALC, alcohol; Alb, albumin; Ht, hematocrit; T-BIL, total bilirubin; Cr, creatinine; BUN, blood urea nitrogen; LC, liver cirrhosis; AIH, autoimmune hepatitis; PBC, primary biliary cirrhosis; SD, standard deviation. 
Table II. Changes in the laboratory values from baseline (day 1) to observation time points.

\begin{tabular}{|c|c|c|c|}
\hline Variables & Day 1 & Day 4 & Day 6 \\
\hline Body weight, kg (n) & 36 & 36 & 36 \\
\hline Mean (SD) & $59.37(15.36)$ & $57.88(15.13)$ & $57.13(14.61)$ \\
\hline Mean change & - & -1.49 & -2.24 \\
\hline 95\% CI (two-sided) & - & $(-2.05,-0.94)$ & $(-3.06,-1.43)$ \\
\hline Serum Alb, g/dl (n) & 38 & 37 & 38 \\
\hline Mean (SD) & $2.29(0.34)$ & $3.00(0.50)$ & $3.26(0.58)$ \\
\hline Mean change & - & 0.70 & 0.97 \\
\hline 95\% CI (two-sided) & - & $(0.59,0.81)$ & $(0.83,1.11)$ \\
\hline $\mathrm{Ht}_{\text {ratio }}$, $\%(\mathrm{n})$ & 38 & 37 & 38 \\
\hline Mean (SD) & $30.23(4.55)$ & $28.75(4.63)$ & $28.97(4.70)$ \\
\hline Mean change & - & 0.95 & 0.96 \\
\hline 95\% CI (two-sided) & - & $(0.93,0.97)$ & $(0.94,0.98)$ \\
\hline $\mathrm{PRC}^{\mathrm{b}}, \mathrm{pg} / \mathrm{ml}(\mathrm{n})$ & 38 & 38 & 38 \\
\hline Geometric mean (SD) & $38.33(6.78,216.59)$ & $26.96(5.30,137.03)$ & $30.77(6.84,138.37)$ \\
\hline Geometric mean fold change $^{c}$ & - & -0.1528 & -0.0953 \\
\hline 95\% CI (two-sided) & - & $(-0.2510,-0.0545)$ & $(-0.1972,0.0066)$ \\
\hline $\mathrm{ALD}^{\mathrm{d}}, \mathrm{pg} / \mathrm{ml}(\mathrm{n})$ & 38 & 38 & 38 \\
\hline Geometric mean (SD) & $90.32(20.68,394.52)$ & $78.67(25.35,244.13)$ & $82.44(28.09,241.97)$ \\
\hline Geometric mean fold change ${ }^{c}$ & - & -0.0599 & -0.0396 \\
\hline 95\% CI (two-sided) & - & $(-0.1925,0.0726)$ & $(-0.1790,0.0998)$ \\
\hline
\end{tabular}

Table III. Change in ascites status over time.

\begin{tabular}{lccc}
\hline Item & Day 1(\%) & Day 4 $(\%)$ & Day 6 (\%) \\
\hline Ascites status $^{\mathrm{a}}$ & & & \\
- & $0(0.0)$ & $2(5.3)$ & $2(5.3)$ \\
+ & $8(21.1)$ & $12(31.6)$ & $21(55.3)$ \\
$2+$ & $18(47.4)$ & $18(47.4)$ & $11(28.9)$ \\
$3+$ & $12(31.6)$ & $6(15.8)$ & $4(10.5)$ \\
Total & $38(100.0)$ & $38(100.0)$ & $38(100.0)$ \\
Change in & & & \\
ascites status & & & \\
-2 & - & $14(36.8)$ & $18(47.4)$ \\
-1 & - & $24(63.2)$ & $15(39.5)$ \\
0 & - & $0(0.0)$ & $1(2.6)$ \\
+1 & - & $38(100.0)$ & $38(100.0)$ \\
Total & - & &
\end{tabular}

ascites status: -, none; + , mild; $2+$, moderate; $3+$, severe.

day 4 (0.70 g/dl; 95\% CI: 0.59-0.81) and on day $6(0.97 \mathrm{~g} / \mathrm{dl}$; 95\% CI: 0.83-1.11) (Table II), whereas the Ht ratio was significantly decreased on day 4 (0.95; 95\% CI: 0.93-0.97) and on day 6 (0.96; 95\% CI: 0.94-0.98) (Table II). The PRC was significantly decreased on day 4 compared to that at baseline (day 1) (geometric mean fold change: $-0.1528,95 \%$ CI: -0.2510 to -0.0545 ) (Table II). The change in ALD was not found to be statistically significant.

Weight loss correlates with the total amount of infused HSA. An exploratory analysis was performed to identify the correlation between weight loss and total dose of HSA, anti-ALD and loop diuretics and on the combined total amount of anti-ALD and loop diuretics. The results revealed that the mean weight loss on day 4 was $-0.29,-0.97$ and -2.13 in the HSA low-dose (HSA $\leq 37.5 \mathrm{~g})$, middle-dose $(37.5<\mathrm{HSA} \leq 60 \mathrm{~g})$ and high-dose group $(60<\mathrm{HSA} \leq 75 \mathrm{~g})$, respectively and the mean weight loss on day 6 was $-0.55,-1.27$ and $-3.24 \mathrm{~kg}$ in the HSA low-dose (HSA $\leq 62.5 \mathrm{~g})$, middle-dose $(62.5<\mathrm{HSA} \leq 100 \mathrm{~g})$ and high-dose group $(100<\mathrm{HSA} \leq 125 \mathrm{~g})$, respectively. This finding suggested that weight loss was dependent on the total amount of HSA on days 4 and 6 (Table IV). Since there are several types of diuretics, each diuretic was classified as anti-ALD or loop diuretics prior to the investigation. The value of each diuretic that was classified as anti-ALD was converted to the value of spironolactone, i.e., $400 \mathrm{mg}$ of potassium canrenoate was equivalent to $100 \mathrm{mg}$ of spironolactone. The value of each diuretic that was classified as a loop diuretic was converted to intravenous furosemide, i.e., $40 \mathrm{mg}$ of oral furosemide and $60 \mathrm{mg}$ of azosemide were equivalent to $20 \mathrm{mg}$ of intravenous furosemide. The results 
Table IV. Mean weight loss by total HSA.

A, Mean weight loss $(\mathrm{kg})$ on day 4 by total HSA

\begin{tabular}{lcccc}
$\begin{array}{l}\text { Total amount of HSA }(\mathrm{g}) \\
(3 \text { days completed })\end{array}$ & No. & Mean & Median & SD \\
\hline HSA $\leq 37.5$ & 8 & -0.29 & 0.05 & 1.00 \\
$37.5<$ HSA $\leq 60$ & 7 & -0.97 & -0.6 & 1.04 \\
$60<$ HSA $\leq 75$ & 21 & -2.13 & -2.0 & 1.70 \\
\hline
\end{tabular}

B, Mean weight loss $(\mathrm{kg})$ on day 6 by total HSA

Total amount of HSA $(\mathrm{g})$

\begin{tabular}{lcccc}
$(5$ days completed $)$ & No. & Mean & Median & SD \\
\hline HSA $\leq 62.5$ & 4 & -0.55 & -0.1 & 1.27 \\
$62.5<$ HSA $\leq 100$ & 7 & -1.27 & -0.8 & 1.95 \\
$100<$ HSA $\leq 125$ & 17 & -3.24 & -2.8 & 2.71
\end{tabular}

HSA, human serum albumin; SD, standard deviation.

Table V. Mean weight loss by total diuretic (anti-ALD).

A, Mean weight loss (kg) on day 4 by total diuretic (anti-ALD)

Total amount of anti-ALD ${ }^{\mathrm{a}}$

\begin{tabular}{lcccc} 
(mg) (3 days completed) & No. & Mean & Median & SD \\
\hline Anti-ALD $=0$ & 3 & 0.10 & 0.1 & 0.30 \\
$0<$ anti-ALD $\leq 150$ & 24 & -1.36 & -1.25 & 1.49 \\
Anti-ALD $>150$ & 9 & -2.39 & -1.9 & 1.81
\end{tabular}

$\mathrm{B}$, Mean weight loss $(\mathrm{kg})$ on day 6 by total diuretic (anti-ALD)

Total amount of anti-ALD

(mg) (5 days completed) No. Mean Median SD

\begin{tabular}{lccrl}
\hline Anti-ALD $=0$ & 3 & 0.27 & 0.3 & 0.15 \\
$0<$ anti-ALD $\leq 250$ & 24 & -2.11 & -1.5 & 2.30 \\
Anti-ALD $>250$ & 9 & -3.42 & -3.2 & 2.49
\end{tabular}

${ }^{a}$ The value of each diuretic that was classified into the anti-ALD group was converted to that of spironolactone, i.e., $400 \mathrm{mg}$ of potassium canrenoate was equivalent to $100 \mathrm{mg}$ of spironolactone. ALD, aldosterone; SD, standard deviation.

demonstrated that weight loss did not clearly depend on the combined total amount of the two types of diuretics, but rather on the total amount of each diuretic on days 4 and 6 (Tables V-VII).

In addition, to identify the factors that affect weight loss, a multiple linear regression analysis was performed in the patients who completed the HSA treatment for 3 and 5 days using an objective variable (weight loss) and explanatory variables (total amount of HSA, anti-ALD and loop diuretic and PRC and ALD values at the initiation of HSA treatment). The
Table VI. Mean weight loss by total diuretic (loop diuretic).

A, Mean weight loss $(\mathrm{kg})$ on day 4 by total diuretic (loop diuretic)

Total amount of

loop diuretic (mg)

(3 days completed) No. Mean Median SD

\begin{tabular}{lcccc}
\hline Loop diuretic $=0$ & 1 & -0.10 & -0.1 & - \\
$0<$ loop diuretic $\leq 60$ & 17 & -1.28 & -0.5 & 1.85 \\
Loop diuretic $>60$ & 18 & -1.77 & -1.85 & 1.42 \\
\hline
\end{tabular}

B, Mean weight loss $(\mathrm{kg})$ on day 6 by total diuretic (loop diuretic)

Total amount of

loop diuretic (mg)

(5 days completed) No. Mean Median SD

\begin{tabular}{lcccc}
\hline Loop diuretic $=0$ & 1 & -0.20 & -0.2 & - \\
$0<$ loop diuretic $\leq 100$ & 16 & -1.70 & -0.7 & 2.66 \\
Loop diuretic $>100$ & 19 & -2.81 & -2.8 & 2.13
\end{tabular}

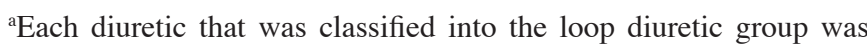
converted to intravenous furosemide, i.e., $40 \mathrm{mg}$ of oral furosemide and $60 \mathrm{mg}$ of azosemide were equivalent to $20 \mathrm{mg}$ of intravenous furosemide. SD, standard deviation.

results demonstrated that only the total amount of HSA was significantly associated with weight loss ( $P R C, P=0.0012$ on day 4 and $\mathrm{P}=0.0229$ on day $6 ; \mathrm{ALD}, \mathrm{P}=0.0016$ on day 4 and $\mathrm{P}=0.0200$ on day 6 ).

\section{Discussion}

HSA treatment is used in patients with diuretic-resistant cirrhotic ascites in order to control the decreased colloidal osmotic pressure due to hypoalbuminemia and to excrete the excess water that was ultimately retained as ascites or edema in the urine. Several factors, including hormones, are considered to be associated with the process of improvement in the colloidal osmotic pressure and the reduction of the ascites. However, the precise mechanism underlying the decrease in the ascites is complicated and has not yet been fully elucidated $(7,9)$. We previously compared the administration of diuretics alone with the combined administration of KD-294 (recombinant HSA) and diuretics, in order to investigate the association between the predicted parameters for the decrease of ascites and weight loss, which was a surrogate marker for the decrease of ascites in the phase II clinical trial of KD-294. As a result, we focused on PRC at the time of initiation of treatment as a factor affecting weight loss $(8,10,11)$.

In this clinical study, the use of diuretics was not restricted and the HSA dose was tailored to each patient's requirements for the treatment of ascites, with the treatment duration set to a maximum of 7 days. Thus, this study was conducted to assess the correlation between each laboratory parameter and weight 
Table VII. Mean weight loss by combined total diuretic (anti-ALD and loop diuretic).

A, Mean weight loss $(\mathrm{kg})$ on day 4 by combined total diuretic (anti-ALD and loop diuretic)

\begin{tabular}{|c|c|c|c|c|c|}
\hline $\begin{array}{l}\text { Total amount of } \\
\text { anti-ALD (mg) } \\
\text { ( } 3 \text { days completed) }\end{array}$ & $\begin{array}{l}\text { Total amount of } \\
\text { loop diuretic (mg) } \\
\text { (3 days completed) }\end{array}$ & No. & Mean & Median & SD \\
\hline \multirow[t]{3}{*}{ Anti-ALD $=0$} & Loop diuretic $=0$ & 0 & - & - & - \\
\hline & $0<$ loop diuretic $\leq 60$ & 2 & -0.05 & -0.05 & 0.21 \\
\hline & Loop diuretic $>60$ & 1 & 0.40 & 0.4 & - \\
\hline \multirow[t]{3}{*}{$0<$ anti-ALD $\leq 150$} & Loop diuretic $=0$ & 1 & -0.10 & -0.1 & - \\
\hline & $0<$ loop diuretic $\leq 60$ & 13 & -1.31 & -0.9 & 1.74 \\
\hline & Loop diuretic>60 & 10 & -1.55 & -1.7 & 1.21 \\
\hline \multirow[t]{3}{*}{ Anti-ALD>150 } & Loop diuretic $=0$ & 0 & - & - & - \\
\hline & $0<$ loop diuretic $\leq 60$ & 2 & -2.35 & -2.35 & 3.61 \\
\hline & Loop diuretic $>60$ & 7 & -2.40 & -1.9 & 1.49 \\
\hline
\end{tabular}

B, Mean weight loss ( $\mathrm{kg}$ ) on day 6 by combined total diuretic (anti-ALD and loop diuretic)

\begin{tabular}{|c|c|c|c|c|c|}
\hline $\begin{array}{l}\text { Total amount of } \\
\text { anti-ALD (mg) } \\
\text { ( } 5 \text { days completed) }\end{array}$ & $\begin{array}{l}\text { Total amount of } \\
\text { loop diuretic (mg) } \\
\text { (5 days completed) }\end{array}$ & No. & Mean & Median & SD \\
\hline \multirow[t]{3}{*}{ Anti-ALD $=0$} & Loop diuretic $=0$ & 0 & - & - & - \\
\hline & $0<$ loop diuretic $\leq 100$ & 2 & 0.35 & 0.35 & 0.07 \\
\hline & Loop diuretic $>100$ & 1 & 0.10 & 0.1 & - \\
\hline \multirow[t]{3}{*}{$0<$ anti-ALD $\leq 250$} & Loop diuretic $=0$ & 1 & -0.20 & -0.2 & - \\
\hline & $0<$ loop diuretic $\leq 100$ & 12 & -1.68 & -0.85 & 2.48 \\
\hline & Loop diuretic $>100$ & 11 & -2.75 & -2.8 & 2.09 \\
\hline \multirow[t]{3}{*}{ Anti-ALD>250 } & Loop diuretic $=0$ & 0 & - & - & - \\
\hline & $0<$ loop diuretic $\leq 100$ & 2 & -3.85 & -3.85 & 4.60 \\
\hline & Loop diuretic $>100$ & 7 & -3.30 & -3.2 & 2.16 \\
\hline
\end{tabular}

ALD, aldosterone; SD, standard deviation.

loss to allow physicians to select the appropriate treatment course for cirrhotic ascites in the clinical setting. The actual doses of HSA were one or two vials per day and a maximum treatment duration of 7 days.

Regarding the change in each laboratory value, the serum Alb concentration was significantly increased and the Ht ratio was significantly decreased on days 4 and 6 following HSA administration, compared to those at baseline. Weight loss, which was a surrogate marker of decreased ascites, was found to be significantly decreased on days 4 and 6 . The increased serum Alb concentration and decreased $\mathrm{Ht}$ ratio were attributed to the improved colloidal osmotic pressure due to the increased Alb concentration following HSA administration, thereby resulting in an increased effective circulating blood volume. Identical results were reported in the phase II clinical trial of KD-294, confirming the reproducibility of the improving effect of HSA on effective circulating blood volume (8).

PRC, on which we focused in light of the results of our previous phase II clinical trial (8), was found to be significantly decreased on day 4 . Although the mean concentration was decreased on day 6 compared to that on day 1 , the change was not statistically significant (geometric mean fold change, -0.0953 ; $95 \%$ CI: -0.1972 to 0.0066$)$. The reason for this finding may be the fact that, from the data on day 4 , all 38 patients received HSA for 3 days, whereas from the data on day 6,8 of the 38 patients discontinued the study on or after day 4 . Five of these 8 patients exhibited an increased PRC on day 6 compared to that on day 4 . Therefore, no significant decrease was observed on day 6 (data not shown). The levels of ALD were not significantly decreased on days 4 and 6 , which was consistent with the results from our previous phase II clinical trial of KD-294 (8).

In addition to the abovementioned results, an exploratory analysis was performed to identify the factors that significantly affect weight loss in patients with cirrhotic ascites. We analyzed the mean weight loss on days 4 and 6 by total HSA, total anti-ALD, total loop diuretics and combined total anti-ALD and loop diuretics, among several other factors that affect weight loss. A dose-dependent increase in the mean weight loss was observed on days 4 and 6 by total HSA, total anti-ALD and total loop diuretics. One of the dose groups 
included only one patient, therefore, the result of anti-ALD and loop diuretics on weight loss was not reliable (Table VII). Thus, it was suggested that the results may not clearly reflect the increase in weight loss dependent on the total administration amount. However, the results generally suggested that there may be a correlation between total diuretic or HSA amount and weight loss.

In addition to these factors, we measured the PRC and ALD levels at the time of HSA administration and performed multiple linear regression analyses to identify the factors affecting weight loss. The results indicated that only total HSA was significantly associated with weight loss. This may have been due to the dose-response of HSA on weight loss, i.e., the efficacy of HSA in patients with cirrhotic ascites was assessed based on the clinical practice data. Therefore, if the patient did not respond adequately after 3 days of HSA administration, the 5-day administration was considered appropriate.

In this clinical study, we were unable to confirm that the PRC at the initiation of HSA administration affects weight loss, as was reported in our KD-294 phase II controlled clinical trial (8). This may have been due to the fact that the targeted population of this study consisted of patients who were co-administered HSA and diuretics. In addition, in the phase II clinical trial, the additional effect of HSA on diuretic therapy was observed in patients with a PRC of $>200 \mathrm{pg} / \mathrm{ml}$ at the initiation of HSA administration. However, the effect of HSA in the group of patients with lower PRC levels $(\leq 200 \mathrm{pg} / \mathrm{ml})$ was not confirmed (8). The mean PRC [139.52 pg/ml, $10^{\text {(Mean-SD); }}$ $\left.35.92 \mathrm{pg} / \mathrm{ml}, 10^{\text {(Mean+SD) }} 541.94 \mathrm{pg} / \mathrm{ml}\right]$ of the patients at the time of the initiation of HSA administration in the phase II clinical trial was higher compared to that of the patients in the present clinical study (8). The mean PRC of the patients in this study was also lower compared to that of the patients in the phase II clinical trial, with a value of $\leq 200 \mathrm{pg} / \mathrm{ml}$ in $\geq 75 \%$ of the patients (29/38) (data not shown). Therefore, the results of the previous and the present study taken together suggest that we may expect HSA administration to decrease ascites in patients with a PRC of $\leq 200 \mathrm{pg} / \mathrm{ml}$ in clinical practice. We were unable to directly compare the results of this study and that of the previous phase II clinical trial, as the physicians were allowed to select the precise combination of HSA and diuretics. However, we consider the conclusion that HSA was not effective due to a low PRC to be rather simplistic.

The multiple linear regression analysis results were not statistically significant. However, due to the fact that the $\mathrm{P}$-value of the loop diuretic was near 0.1, significant results may be dependent on sample size. We hypothesized that the total loop diuretics may be a significant factor, next to total HSA, in the combination therapy of HSA and diuretics.

The dosage of HSA used in this study was within the recommended range of actual dosages used in the medical setting. As a clearly significant effect of HSA was demonstrated in the multiple linear regression analysis of the 3-day administration (the shortest allowed treatment duration), we consider the observed dose-response of HSA reported in this study to be reliable. However, the prescription of diuretics based on a patient's condition may affect the result. A limitation of this study was that its statistical power was low due to the small sample size. Thus, clinical studies with larger sample sizes are required to reconfirm the efficacy of HSA.

\section{Acknowledgements}

This observational study was conducted upon request from Kaketsuken (The Chemo-Sero-Therapeutic Research Institute, Kumamoto, Japan). The authors would like to thank Mr Fujio Matsuo of Statcom Company Ltd. for his statistical advice.

\section{References}

1. Uemura M, Yamano J and Fukui H: The condition and treatment of intractable ascites. Shoukakika (Gastroenterology) 35: 448-458, 2002 (In Japanese).

2. Clinical practice guidelines for the management of liver cirrhosis. Nankodo, Tokyo, 116-149, issued April 25, 2010.

3. Guideline for blood product use and blood transfusion therapy practice. 'Yakusyokuhatsu Notification no. 0726002', July 26, 2007.

4. Runyon BA: Management of adult patients with ascites due to cirrhosis. Hepatology 39: 841-856, 2004.

5. Sort P, Navasa M, Arroyo V, et al: Effect of intravenous albumin on renal impairment and mortality in patients with cirrhosis and spontaneous bacterial peritonitis. N Engl J Med 341: 403-409, 1999.

6. Ortega R, Gines P, Uriz J, et al: Terlipressin therapy with and without albumin for patients with hepatorenal syndrome: results of a prospective, nonrandomized study. Hepatology 36: 941-948, 2002.

7. Gentilini P, Casini-Raggi V, Di Fiore G, et al: Albumin improves the response to diuretics in patients with cirrhosis and ascites: results of a randomized, controlled trial. J Hepatol 30: 639-645, 1999.

8. Nakamura T, Sata M, Suzuki K, et al: Open-labeled randomized controlled trial to compare diuretic therapy with recombinant human serum albumin and diuretic therapy for therapeutic treatment of ascites in patients with advanced liver cirrhosis: An exploratory trial. Hepatol Res: Apr 22, 2013 (Epub ahead of print). doi: 10.1111/hepr.12142.

9. Fogel MR, Sawhney VK, Neal EA, Miller RG, Knauer CM and Gregory PB: Diuresis in the ascitic patient: a randomized controlled trial of three regimens. J Clin Gastroenterol 3 (Suppl 1): 73-80, 1981

10. Brinch K, Moller S, Bendtsen F, Becker U and Henriksen JH: Plasma volume expansion by albumin in cirrhosis. Relation to blood volume distribution, arterial compliance and severity of disease. J Hepatol 39: 24-31, 2003.

11. Wong PY, Carroll RE, Lipinski TL and Capone RR: Studies on the renin-angiotensin-aldosterone system in patients with cirrhosis and ascites: effect of saline and albumin infusion. Gastroenterology 77: 1171-1176, 1979. 